\title{
Atropine injection followed by coronary artery spasm with ventricular tachycardia during spinal anesthesia -A case report-
}

\author{
Joon-Ho Lee, Ji-Hye Seok, Young-Lok Kim, Ji-Hyang Lee, Sang-Gon Lee, Eun-Ju Kim, and Da-Mi Seo \\ Department of Anesthesiology and Pain Medicine, Daegu Fatima Hospital, Daegu, Korea
}

Bradycardia may occur during spinal anesthesia with atropine commonly used as a treatment. A 44-year-old female with no known history of any underlying diseases, developed a coronary spasm following ventricular tachycardia when $0.5 \mathrm{mg}$ of atropine was injected intravenously to treat bradycardia during spinal anesthesia. The imbalance caused by atropine in the sympathovagal activity may predispose the coronary artery to develop spasms with ventricular tachycardia. Therefore prudent use of atropine should be accompanied by close monitoring. (Korean J Anesthesiol 2013; 65: 66-70)

Key Words: Atropine, Bradycardia, Coronary artery spasm, Spinal anesthesia, Ventricular tachycardia.

Spinal anesthesia has long been considered a relatively safe technique. However, bradycardia may occasionally occur during spinal anesthesia. Sudden and severe bradycardia and asystolic cardiac arrest are considered to be infrequent, but they are certainly the most serious of complications in spinal anesthesia.

Atropine, an anti-muscarinic drug, is commonly used for the treatment of bradyarrythmias.

Thus, we report one such case of a patient experiencing a coronary spasm following ventricular tachycardia (VT) after atropine during spinal anesthesia.

\section{Case Report}

A 44-year-old female, height and weight of $158 \mathrm{~cm}$; $53 \mathrm{~kg}$, was scheduled for a varicosectomy and a communicating vein ligation of the bilateral varicose veins.

The patient's family history, past history, obstetric history, and social history were unremarkable.

Upon admission, vital signs were stable with a normal physical examination. The electrocardiogram (ECG) displayed a normal sinus rhythm at 57 beats per minute at normal intervals (Fig. 1).

The patient's vital signs on arrival to the operation room showed a blood pressure of 134/72 $\mathrm{mmHg}$ and heart rate of 9093 beats/min.

With the patient in the lateral decubitus position, spinal anesthesia was administered using a 25-gauge Quincke-type needle with $12 \mathrm{mg}$ of bupivacaine (Marcaineheavy ${ }^{\circledR}$ ) at the L3L4 interspace.

Received: August 14, 2012. Revised: September 10, 2012. Accepted: September 12, 2012.

Corresponding author: Ji-Hyang Lee, M.D., Department of Anesthesiology and Pain Medicine, Daegu Fatima Hospital, 576-31, Sinam-dong, Dong-gu, Daegu 701-600, Korea. Tel: 82-53-940-7434, Fax: 82-53-954-7417, E-mail: lovehan3@yahoo.co.kr

(c) This is an open-access article distributed under the terms of the Creative Commons Attribution Non-Commercial License (http:// creativecommons.org/licenses/by-nc/3.0/), which permits unrestricted non-commercial use, distribution, and reproduction in any medium, provided the original work is properly cited. 
The patient was placed in the supine position for 10 minutes, where the blood pressure lowered to $120 / 70 \mathrm{mmHg}$ and the heart rate decreased to $82-85$ beats $/ \mathrm{min}$. The maximal level of the sensory block to cold temperature was T8.

Thereafter, $2.5 \mathrm{mg}$ of midazolam were given intravenously for the sedation and resolution of anxiety.

The patient's blood pressure was then stabilized to $110^{-90 / 65-}$ $45 \mathrm{mmHg}$. After one hour since the start of the operation, the patient's heart rate began to gradually decrease. However, since the patient's blood pressure was stable and she was successfully sedated, we decided to observe and no supplementary evaluation of block level was performed. Two hours after the administration of the spinal anesthesia, the heart rate dropped to $42-$ 45 beats/min, and $0.5 \mathrm{mg}$ of atropine was injected intravenously. The patient's heart rate rose to 60 beats per minute within
5 minutes. Shortly after, premature ventricular contraction occurred in the ECG followed by ventricular tachycardia, where the patient's heart rate increased to 140 beats per minute and the blood pressure to $120 / 60 \mathrm{mmHg}$ (Fig. 2 and 3). The patient complained of chest discomfort, but was capable of communication and cooperation. At that time, the upper sensory block level to cold temperature was T10. The patient was given $10 \mathrm{mg}$ of esmolol intravenously. After 2-3 minutes, the patient's ECG presented an elevation in the ST with a heart rate of 62 beats/min (Fig. 4). After the termination of the operation, the patient was transferred to the intensive care unit. Laboratory tests at the moment of ventricular tachycardia were nonspecific. The CPK-MB was $19.7 \mathrm{ng} / \mathrm{ml}$; and Troponin I peaked at $6.38 \mathrm{ng} / \mathrm{ml}$, where both subsequently returned back to normal within 2 days. Thyroid function test and fasting lipid

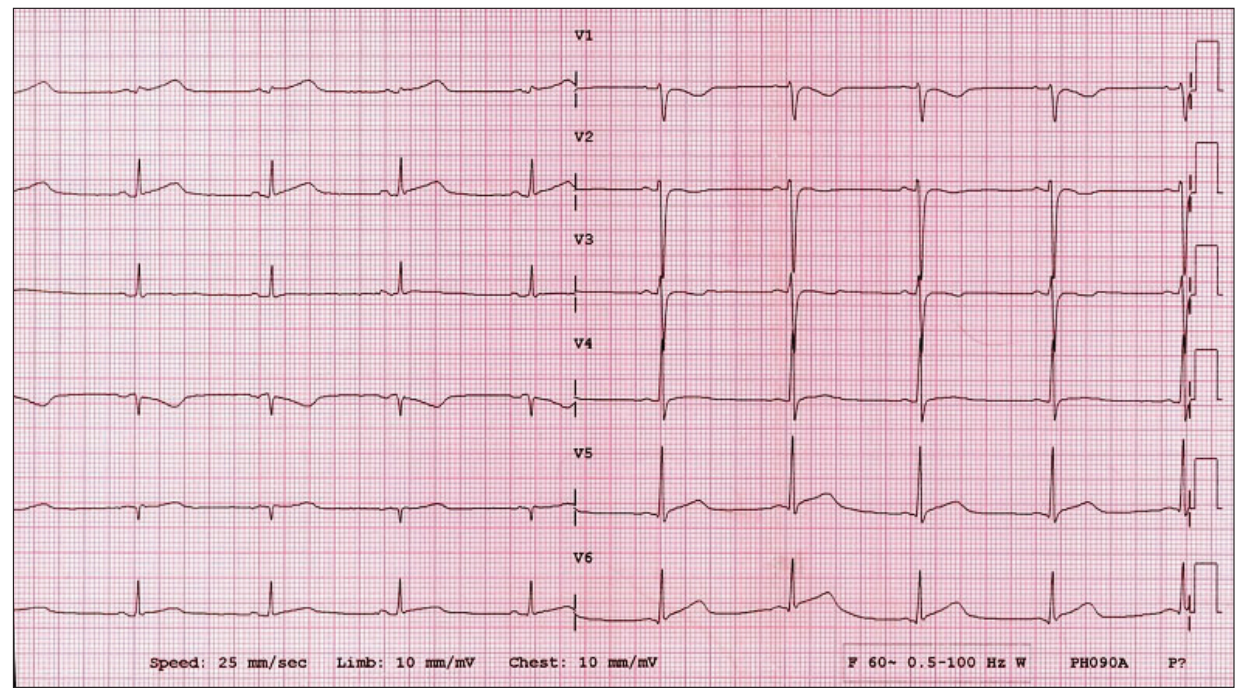

Fig. 1. Preoperative electrocardiogram shows normal sinus rhythm.



Fig. 2. Premature ventricular complexes and ventricular tachycar appearing five minutes after the administration of atropine. 

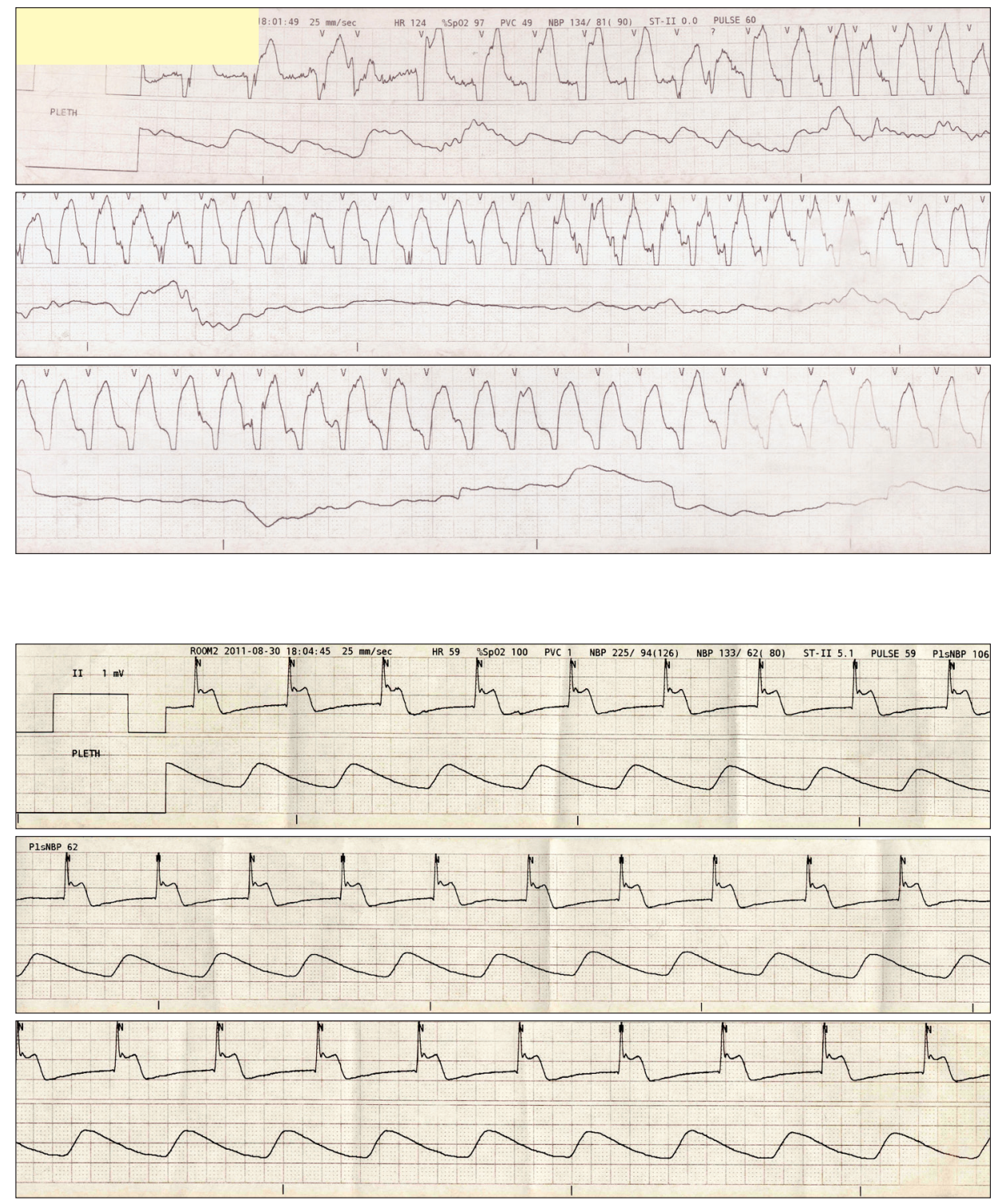

Fig. 3. Electrocardiogram showing ventricular tachycardia. profile were within normal limits.

Coronary angiography (CAG) that was performed after the operation showed luminal stenosis of up to $40 \%$ at the middle left anterior descending coronary artery (mLAD), while left circumflex artery (LCX) and right coronary artery (RCA) showed no significant signs of luminal stenosis. Ultrasonic cardiography (UCG) that was performed after the operation showed an ejection fraction of $40 \%$, akinesia at the LCX territory with mild left ventricle (LV) systolic dysfunction, and impaired relaxation. According to the CAG and UCG results that were obtained by the consultation of the department of cardiology, the patient was diagnosed with VT due to vasospasm. After the administration of calcium channel blockers, the patient was discharged 3 days later without any further symptoms.

\section{Discussion}

Although spinal anesthesia has long been considered a safe technique, it is known to have several side effects - such as hypotension, bradycardia, dysrhythmia, nausea and vomiting. Carpenter et al reported that the incidence of bradycardia (heart rate $<60$ beats/min) during spinal anesthesia (SA) is $13 \%$, with variables conferring to increased odds of developing bradycardia include a baseline heart rate $<60$ beats/min (odds ratio $4.9, \mathrm{P}<$ $0.001)$, the ASA physical status status classification of 1 versus 3 or 4 (3.5, $\mathrm{P}<0.001)$, current therapy with $\beta$-adrenergic blocking drugs $(2.9, \mathrm{P}<0.001)$, and peak block height $\geq \mathrm{T} 5(1.7, \mathrm{P}=0.02)$. Three simple factors (baseline heart rate, ASA physical status, and $\beta$-blockade) have a stronger correlation than the peak block 
height. These findings suggest that the imbalance between the sympathetic and parasympathetic tone may play an important role in the susceptibility to the development of bradycardia during spinal anesthesia [1]. Abrupt, extreme bradycardia and/ or asystolic cardiac arrest during spinal anesthesia seldom occurs, but can be life-threatening when they appear [2]. In order to prevent such side effects, atropine is used to treat bradycardia with heartbeat rates below 50 beats/min $[3,4]$. In our patient, atropine was injected intravenously when the patient's heart rate was between $42-45$ beats/min, although the blood pressure was stable.

Atropine, a potent cardiac parasympatholytic blocking agent, increases the heart rate and is widely used to treat bradycardia. There are three distinct phases of atropine action on the heart, which include an initial vagotonic effect, a transient period of vagal imbalance at different levels of the conduction system, and a final prolonged period of parasympathetic blockage [5].

The influence of atropine on the autonomic nervous system which controls the heart is studied via spectral analysis of the heart rate variability (HRV). The spectral analysis of the HRV can distinguish the parasympathetic from the sympathetic influence to the heart. The low frequency (LF) component of the spectral HRV reflects the sympathetic activity with some influence from the vagal activity, whereas the high frequency (HF) component is almost exclusively mediated by the vagal activity. The LF/HF ratio has been used as an index for the sympathovagal balance of the heart, with its increase suggesting sympathetic predominance as a result of increased sympathetic or decreased parasympathetic drive to the heart, or a combination of both [6]. In a report of atropine-induced ventricular tachycardia, atropine is thought to initially decrease parasympathetic activity and then provoke unopposed sympathetic activity [7]. This enhanced sympathetic activity probably contributed to the provocation of the coronary artery spasm in our case.

The coronary arteries are innervated by both sympathetic and parasympathetic neurons, and alterations to either nervous system may cause coronary artery spasms. There are many factors involved in the occurrence of perioperative coronary artery spasms - activated sympathetic activity, activated parasympathetic activity, alkalosis, and calcium drugs, etc. Spinal anesthesia by itself can be the cause of coronary artery spasms. It has been shown that a spinal or extradural blockade denervates the sympathetic nervous system by two to three segments above the level of the somatic block. Above the level of the sympathetic blockade, compensatory vasoconstriction is observed, presumably in an effort to offset the reduction in the peripheral resistance caused by the block. This sympathetic activity reflex involves the cardiac sympathetic nerves causing coronary vasoconstriction and the consequent ischemia [8]. It is likely, in this current case, that a combination of an activated sympathetic nervous system by atropine and spinal anesthesia may be responsible for the coronary artery spasm. Therefore, anesthesiologists should be aware that atropine may provoke coronary artery spasms, especially during spinal anesthesia.

There are some reports of ventricular tachycardia that are induced by atropine in the presence of significant ischemic heart disease $[9,10]$. These reports warned of the side effects of atropine administration in patients with ischemic myocardium. In this case, an absence of a preoperative diagnosis of ischemic heart disease (IHD) allowed us to administer atropine. However, as postoperative evaluation showed that the results suggested IHD, the susceptibility to coronary spasms through atropine may be relatively high for this patient.

Although there are currently no established guidelines for the treatment of bradycardia, it is widely believed that a heart rate below 50 beats per minute after the induction of SA requires treatment. However, further considerations should be taken in regards to the benefits found from the use of atropine in bradycardia, which is not accompanied by hypotension. As in this case, administration of atropine in patients with previously undiagnosed IHD may cause adverse outcomes, such as coronary spasms or ventricular arrhythmia. Therefore, it appears that the administration of atropine should be used with caution and on a flexible basis when bradycardia without hypotension occurs after SA during clinical practice.

\section{References}

1. Carpenter RL, Caplan RA, Brown DL, Stephenson C, Wu R. Incidence and risk factors for side effects of spinal anesthesia. Anesthesiology 1992; 76: 906-16.

2. Løvstad RZ, Granhus G, Hetland S. Bradycardia and asystolic cardiac arrest during spinal anaesthesia: a report of five cases. Acta Anaesthesiol scand 2000; 44: 48-52.

3. Park SK, Kim YK, Chung SL, Chin JH, Lee C, Lee YM. Effects of Patients' Position on blood pressure and heart rate during spinal anesthesia for Axillo-femoral bypass surgery. Korean J Anesthesiol 2006; 51: 675-9.

4. Kim SH, Cho MJ, Shin YS, Lee JS, Lee JW, Yoon KB. Spinal anesthesia with $3.75 \mathrm{mg}$ of $0.25 \%$ hyperbaric bupivacaine for diabetic foot surgery. Korean J Anesthesiol 2009; 56: 273-9. 
5. Averill KH, Lamb LE. Less commonly recognized actions of atropine on cardiac rhythm. Am J Med Sci 1959; 237: 304-18.

6. Hayashi H, Fujiki A, Tani M, Mizumaki K, Shimono M, Inoue H. Role of sympathovagal balance in the initiation of idiopathic ventricular tachycardia originating from the right ventricular outflow tract. Pacing Clin Electrophysiol 1997; 20: 2371-7.

7. Gujja K, Pai VM, Aslam AF, Saponieri C, Vasavada B. Atropine-induced polymorphic ventricular tachycardia: a rare and bygone occurrence revisited. Am J Ther 2010; 17: e175-8.

8. Krantz EM, Viljoen JF, Gilbert MS. Prinzmetal's variant angina during extradural anaesthesia. Br J Anaesth 1980; 52: 945-9.

9. Massumi RA, Mason DT, Amsterdam EA, DeMaria A, Miller RR, Scheinman MM, et al. Ventricular fibrillation and tachycardia after intravenous atropine for treatment of bradycardias. N Engl J Med 1972; 287: 336-8.

10. Goel VK, Mehrotra TN, Gupta SK. Ventricular tachyarrhythmias: complication of atropine therapy in acute myocardial infarction. Indian Heart J 1981; 33: 301-2. 